\title{
Разработка Алгоритмов и Программных Средств для Выявления Неисправностей Технических Объектов на Ранней Стадии
}

\author{
Тельман Алиев ${ }^{1}$, Наиля Мусаева ${ }^{2}$, Матанат Сулейманова $^{3}$ \\ ${ }^{1,3}$ Институт Систем Управления НАНА, Баку, Азербайджан \\ ${ }^{2}$ Азербайджанский Университет Архитектуры и Строительства, Баку, Азербайджан \\ ${ }^{1}$ telmancyber@gmail.com, ${ }^{2}$ musanaila@gmail.com, ${ }^{3}$ metanet_suli@yahoo.com
}

\begin{abstract}
Аннотация- Разработаны алгоритмы и технологии вычисления характеристик помехи, которая зарождается в результате появления неисправности в техническом состоянии объекта. Разработаны программные средства вычисления этих характеристик. Показано, что применение данных пакетов программ в системах мониторинга, контроля, диагностики, управления и т.д. позволяет выявить неисправность на ранней стадии, и, таким образом, предотвратить возникновение аварийных ситуаций.
\end{abstract}

Ключевые слова- помеха, неисправность, пакет программ, мониторинг

\section{I. ВВЕДЕНИЕ}

Известно, что при работе систем мониторинга, контроля, диагностики, прогноза, идентификации, управления и т.д. на технологические параметры $X(t)$ действуют различные помехи $\mathrm{E}(t)$, под действием которых происходит искажение сигналов:

$$
G(t)=X(t)+\mathrm{E}(t) .
$$

Это внутренняя помеха, которая возникает в результате изменения технического состояния исследуемого объекта вследствие появления дефектов, износов, коррозии, трещин, поломок и т.д. Например, при эксплуатации штанговых насосов помехи появляются от зарождения таких неисправностей, как прихват плунжера, утечка нагнетательного клапана (УНК), утечка нагнетательного клапана (УНК) и труб, утечка приемного клапана (УПК), течь в насосных трубах, ослабление, приводящее к обрыву штанг. Для объектов теплоснабжения помехи появляются в результате таких неисправностей, как изношенность котельного оборудования; повреждения, возникающие на действующих газопроводах; повреждение тепловых сетей, связанные с образованием наружной коррозии трубопроводов; коррозионное разрушение металлов трубопровода в водной среде; потеря напора трубопровода от утечки воды; накипные отложения в теплообменниках и т.д. $[1,2]$.

Из всех перечисленных неисправностей самыми опасными неисправностями являются те, которые могут привести к аварийным ситуациям, а также к необходимости проведения капитального ремонта и остановки работы оборудования. Самыми безопасными являются те, устранение которых сводится всего лишь к локализации места образования дефекта. А это возможно только в том случае, если определить дефект на ранней стадии. Существующие методы выявления дефектов сводятся к техническому решению этой проблемы, когда дефект приобретает явно выраженную форму [3-5]. В работах же [6-10] показано, что вычисление значений характеристик помехи, которая появляется в результате возникновения дефекта, позволяет на ранней стадии установить наличие неисправности в техническом состоянии исследуемого объекта. Причем по значению среднего квадратического отклонения помехи можно выявить момент зарождения дефекта, а по функции плотности распределения можно выявить степени развития этой неисправности. Использование алгоритмов, технологий и программных средств вычисления характеристик помехи в системах мониторинга и контроля позволит выявить неисправность технических объектов на ранней стадии и своевременно провести соответствующие ремонтные работы.

\section{II. ПОСТАНОВКА ЗАДАЧИ}

На временном интервале $0 \leq t \leq T$ наблюдается непрерывный случайный стационарный эргодический зашумленный технологический процесс $G(t)$, состоящий из суммы случайной полезной составляющей $X(t)$ и случайной помехи $\mathrm{E}(t)$, которые также являются стационарными эргодическими и их невозможно выделить из $G(t)$. Случайный процесс $G(t)$ может подчиняться различным законам распределения и для него можно вычислить выборочные оценки таких характеристик как математическое ожидание $m_{G}$, дисперсию $D_{G}$, среднее квадратическое отклонение $\sigma_{G}$, корреляционную функцию $R_{G G}(\tau)$ по формулам [11-12]:

$$
m_{G}=\frac{1}{N} \sum_{i=1}^{N} G(i \Delta t)
$$




$$
\begin{gathered}
D_{G}=\frac{1}{N} \sum_{i=1}^{N}\left(G(i \Delta t)-m_{G}\right)^{2}=\frac{1}{N} \sum_{i=1}^{N} \stackrel{\circ}{G}(i \Delta t), \\
\sigma_{G}=\sqrt{D_{G}}, \\
R_{\substack{G G}}(\mu)=\frac{1}{N} \sum_{i=1}^{N} \stackrel{\circ}{G}(i \Delta t) \stackrel{\circ}{G}((i+\mu) \Delta t)
\end{gathered}
$$

где $\stackrel{\circ}{G}(t)=G(t)-m_{G}, \tau=0, \quad \Delta t, \quad 2 \Delta t, \quad 3 \Delta t, \quad \ldots$. временной сдвиг.

Известно, что в системе мониторинга, контроля, диагностики, прогноза, управления, идентификации и т.д. помеха появилась в результате возникновения дефектов, неисправностей, неполадок и т.д., имеет нормальное распределение $N\left(\varepsilon ; m_{\varepsilon}, \sigma_{\varepsilon}\right)$ и нулевое среднее $m_{\varepsilon}=0$. Так как стационарная случайная помеха $\mathrm{E}(t)$ является эргодической, то ее математическое ожидание $m_{\mathrm{E}}$ и среднее квадратическое отклонение $\sigma_{\mathrm{E}}$ имеют одно и то же значение для любой из случайных функций, входящих в совокупность. Поэтому функцию плотности нормального распределения $N\left(\varepsilon ; m_{\mathrm{E}}, \sigma_{\mathrm{E}}\right)=N(\varepsilon)$ помехи представим в виде [11-12]:

$$
N(\varepsilon)=\frac{1}{\sigma_{\mathrm{E}} \sqrt{2 \pi}} e^{-\frac{\left(\varepsilon-m_{\varepsilon}\right)^{2}}{2 \sigma_{\mathrm{E}}^{2}}} .
$$

Из этой формулы очевидно, что для определения функции плотности распределения $N(\varepsilon)$ помехи $\mathrm{E}(t)$, необходимо знание среднего квадратического отклонения $\sigma_{\mathrm{E}}$, которое неизвестно, и его значение невозможно выделить из зашумленного процесса $G(t)$. Поэтому ниже предлагается алгоритм и пакет программ вычисления среднего квадратическогоотклонения $\sigma_{\mathrm{E}}$ помехи $\mathrm{E}(t)$ и использования его для определения дискретных значений функции плотности нормального распределения $N(\varepsilon)$ помехи.

\section{III. АЛГОРИТМ ВЫЧИСЛЕНИЯ ДИСКРЕТНЫХ ЗНАЧЕНИЙ ФУНКЦИИ ПЛОТНОСТИ НОРМАЛЬНОГО РАСПРЕДЕЛЕНИЯ ПОМЕХИ}

Пусть от датчика, размещенного в зоне действия влияющих на объект факторов, поступают цифровые значения аддитивного зашумленного цифрового сигнала $G(\Delta t)$, состоящего из полезного сигнала $X(\Delta t)$ и помехи $\mathrm{E}(\Delta t)$. Шаг дискретизации $\Delta t$ выбранным в соответствии с условием: $\Delta t=1 / 2 \omega_{\mathrm{E}}$, где $\omega_{\mathrm{E}}-$ частота среза помехи [11-12]:

Тогда алгоритм вычисления дискретных значений функции плотности распределения $N^{*}(\varepsilon)$ помехи $\mathrm{E}(\Delta t)$ можно представить следующим образом [6-10]:

1) Вычисляются дискретные оценки авто корреляционной функции центрированного зашумленного сигнала $G(\Delta t)$ при $\mu=0, \Delta t, 2 \Delta t$ :

$$
\begin{gathered}
R_{G G}^{\circ \circ}(\mu=0)=\frac{1}{N} \sum_{i=1}^{N} \stackrel{\circ}{G}(i \Delta t) \stackrel{\circ}{G}(i \Delta t), \\
R_{\substack{G G \\
R^{\circ}}}(\mu=\Delta t)=\frac{1}{N} \sum_{i=1}^{N} \stackrel{\circ}{G}(i \Delta t) \stackrel{\circ}{G}((i+1) \Delta t), \\
R_{G G}^{\circ}(\mu=2 \Delta t)=\frac{1}{N} \sum_{i=1}^{N} \stackrel{\circ}{G}(i \Delta t) \stackrel{\circ}{G}((i+2) \Delta t) .
\end{gathered}
$$

2) Вычисляется среднее квадратическоеотклонение $\sigma_{\mathrm{E}}^{*}$ помехи $\mathrm{E}(t)$ зашумленного сигнала $G(t)$ [3-10]:

$$
\sigma_{\mathrm{E}}^{*}=\sqrt{D_{\mathrm{E}}^{*}}
$$

где

$$
D_{\mathrm{E}}^{*}=R_{G G}(\mu=0)-2 R_{G G}(\mu=\Delta t)+R_{G G}(\mu=2 \Delta t) .
$$

3) $\mathrm{C}$ определенным шагом $\Delta \varepsilon$ задается последовательность возможных значений помехи $\mathrm{E}(t)$ в порядке возрастания от $\varepsilon_{\min }$ до $\varepsilon_{\max }$ :

$$
\varepsilon(1)=\varepsilon_{\min }, \varepsilon(i+1)=\varepsilon(i)+\Delta \varepsilon, \ldots, \varepsilon_{\max },
$$

и формируется последовательность возможных значений помехи

$$
\varepsilon(1), \varepsilon(2), \varepsilon(3), \ldots, \varepsilon_{\max },
$$

для которой выполняется условие $\varepsilon(i-1)<\varepsilon(i)$.

Затем в точках $\varepsilon(1), \varepsilon(2), \varepsilon(3), \ldots, \varepsilon_{\max }$ вычисляется функция плотности нормального распределения помехи $\mathrm{E}(t)$. Учитывая , что $m_{\varepsilon}=0$, получаем

$$
N^{*}(\varepsilon(i))=\frac{1}{\sigma_{\mathrm{E}}^{*} \sqrt{2 \pi}} e^{-\frac{\varepsilon^{2}(i)}{2\left(\sigma_{\mathrm{E}}^{*}\right)^{2}}}
$$

Вычисляется максимум функции плотности распределения $N_{\max }(\varepsilon)$ помехи $\mathrm{E}(t)$ зашумленного сигнала $G(t)$ :

$$
N_{\max }^{*}(\varepsilon=0)=\frac{1}{\sigma_{\mathrm{E}}^{*} \sqrt{2 \pi}} .
$$


4) Вычисляются координаты точек перегиба $\left(-\sigma_{\mathrm{E}}^{*} ; \frac{1}{\sigma_{\mathrm{E}}^{*} \sqrt{2 \pi e}}\right)$ и $\left(\sigma_{\mathrm{E}}^{*} ; \frac{1}{\sigma_{\mathrm{E}}^{*} \sqrt{2 \pi e}}\right)$ функции плотности распределения помехи:

- для первой точки по оси абсцисс:

$$
A \mathrm{E} 1=-\sqrt{\sigma_{\mathrm{E}}^{*}}
$$

- для второй точки по оси абсцисс:

$$
A \mathrm{E} 2=\sqrt{\sigma_{\mathrm{E}}^{*}}
$$

- для первой и второй точек по оси ординат:

$$
O \mathrm{E}=\frac{1}{\sqrt{2 \sigma_{\mathrm{E}}^{*} \pi e}} .
$$

IV. ОПИСАНИЕ ПАКЕТА ПРОГРАММ ДЛЯ

ВЫЧИСЛЕНИЯ СРЕДНЕГО КВАДРАТИЧЕСКОГО ОТКЛОНЕНИЯ И ФУНКЦИИ ПЛОТНОСТИ РАСПРЕДЕЛЕНИЯ ПОМЕХИ

Программы вычисления среднего квадратического отклонения и функции плотности распределения помехи написаны с использованием средства компьютерной математики MATLAB. Пакет программ состоит из следующих блоков.

1. Вводятся дискретные значения $G(i \Delta t)$ исследуемого сигнала.

2. Вычисляются дискретные оценки авто корреляционной функции центрированного зашумленного сигнала $G(\Delta t)$ при $\mu=0, \Delta t, 2 \Delta t$ :

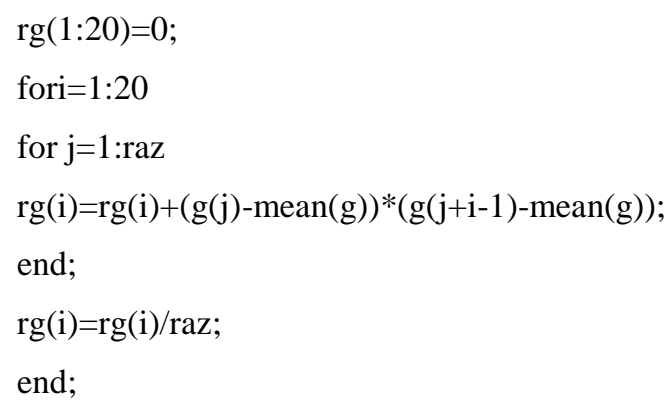

3. Вычисляется дисперсия $D_{\mathrm{E}}^{*}$ помехи:

$$
d \times 1=r g(1)+r g(3)-2 * r g(2) ;
$$

4. Вычисляется среднее квадратическоеотклонение $\sigma_{\mathrm{E}}^{*}$ помехи:

$$
\operatorname{sdx} 1=\operatorname{sqrt}(\mathrm{dx} 1)
$$

5. Вычисляется функция плотности распределения помехи $\mathrm{E}(t)$ :

vmue $=0$;

ve1=vmue- $3 * \operatorname{sdx} 1: 1:$ vmue $+3 * \operatorname{sdx} 1$; vye $=$ normpdf $(\mathrm{e} 1, \mathrm{vmue}, \mathrm{sdx} 1)$;

6. Вычисляется максимум функции плотности распределения помехи $\mathrm{E}(t)$ :

vyemax $=\max ($ vye $)$;

vyemaxv $=1 /\left(\operatorname{sdx} 1 *(2 * \mathrm{pi})^{\wedge}(1 / 2)\right)$;

7. Вычисляются координаты точек перегиба функция плотности распределения помехи:

vtpe1=vmue-sdx 1 ;

vtpe $2=$ vmue + sdx 1 ;

vtpye $=1 /\left(\operatorname{sdx} 1 *\left(2 * p^{*} * \exp (1)\right)^{\wedge}(1 / 2)\right)$;

V. АЛГОРИТМЫ

ВЫЯВЛЕНИЯ

НЕИСПРАВНОСТЕЙ ТЕХНИЧЕСКИХ ОБЪЕКТОВ НА РАННЕЙ СТАДИИ С ПОМОЩЬЮ ХАРАКТЕРИСТИК ПОМЕХИ

Ниже предлагается алгоритмы и технологии выявления неисправностей технических объектов на ранней стадии с помощью характеристик помехи.

1. В момент времени $t_{1}$ вычисляются значения дисперсии помехи $D_{\mathrm{E}_{t 1}}^{*}, \quad$ среднеквадратического отклонения $\sigma_{\mathrm{E}_{t 1}}^{*}$, значений функции плотности распределения $N_{t 1}^{*}(\varepsilon(i))$, ее максимума $N_{t 1, \max }^{*}(0)$ и точек перегиба по оси абсцисс $A 1_{t 1}, A 2_{t 1}$ и ординат $O_{t 1}$; полученные значения заносятся в банк данных.

2. Затем в момент времени $t_{2}$ вычисляются значения дисперсии помехи $D_{\mathrm{E}_{t 2}}^{*}$, средне квадратического отклонения $\sigma_{\mathrm{E}_{t 2}}^{*}$, значений функции плотности распределения $N_{t 2}^{*}(\varepsilon(i))$, ее максимума $N_{t 2, \max }^{*}(0)$ и точек перегиба по оси абсцисс $A 1_{t 2}, A 2_{t 2}$ и ординат $O_{t 2}$ ; полученные значения также заносятся в банк данных.

3. Проводится анализ оценок помехи, полученных в моменты времени $t_{1}$ и $t_{2}$, и делаются следующие выводы.

3.1. Если

$$
\begin{gathered}
D_{\mathrm{E}_{t 1}}^{*}=D_{\mathrm{E}_{t 2}}^{*}, \sigma_{\mathrm{E}_{t 1}}^{*}=\sigma_{\mathrm{E}_{t 2}}^{*}, \\
N_{t 1}^{*}(\varepsilon(i))=N_{t 2}^{*}(\varepsilon(i)), \\
N_{t 1, \text { max }}^{*}(0)=N_{t 2, \text { max }}^{*}(0), \\
A 1_{t 1}=A 1_{t 2}, A 2_{t 1}=A 2_{t 2}, O_{t 1}=O_{t 2},
\end{gathered}
$$

то динамика развития неисправности не наблюдается. Поэтому можно проводить профилактические работы в режиме нормальной эксплуатации объекта. 


\section{2. Если}

$$
\begin{gathered}
D_{\mathrm{E}_{t 1}}^{*} \neq D_{\mathrm{E}_{t 2}}^{*}, \sigma_{\mathrm{E}_{t 1}}^{*} \neq \sigma_{\mathrm{E}_{t 2}}^{*}, \\
N_{t 1}^{*}(\varepsilon(i)) \neq N_{t 2}^{*}(\varepsilon(i)), \\
N_{t 1, \max }^{*}(0) \neq N_{t 2, \text { max }}^{*}(0), \\
A 1_{t 1} \neq A 1_{t 2}, A 2_{t 1} \neq A 2_{t 2}, O_{t 1} \neq O_{t 2},
\end{gathered}
$$

то неисправность находится в процессе развития. Причем, если

$$
D_{\mathrm{E}_{t 1}}^{*}>D_{\mathrm{E}_{t 2}}^{*}, \sigma_{\mathrm{E}_{t 1}}^{*}>\sigma_{\mathrm{E}_{t 2}}^{*},
$$

то это означает, что дефект увеличился и следует провести ремонтные работы.

\section{3. Если}

$$
D_{\mathrm{E}_{t 1}}^{*} \gg D_{\mathrm{E}_{t 2}}^{*}, \sigma_{\mathrm{E}_{t 1}}^{*} \gg \sigma_{\mathrm{E}_{t 2}}^{*},
$$

то это означает, что повреждение существенно, и неисправность развивается интенсивно. Тогда необходимо остановить работу оборудования во избежание возникновение аварии.

\section{ЗАКЛЮЧЕНИЕ}

Разработаны алгоритмы и программные средства для выявления неисправностей технических объектов на ранней стадии с помощью таких характеристик помехи как дисперсия, среднеквадратическое отклонение, функция плотности распределения, ее максимум, точки перегиба по оси абсцисс и ординат. Показано,что в зависимости от степени изменения значений этих характеристик можно судить о возникновении и интенсивности развития дефекта. Применение разработанных алгоритмов и программных средств в системах мониторинга, контроля, диагностики, управления и т.д. позволяет определить момент, когда необходимо провести соответствующий ремонт, и, таким образом, избежать возникновение аварийных ситуаций.

\section{ЛИТЕРАТУРА}

[1] Установка штангового глубинного насоса (УШГН): http://vseonefti.ru/upstream/shtangovyi-nasos.htm

[2] Новые технологии в теплоснабжении и строительстве: Сборник работ аспирантов и студентов - сотрудников научноисследовательской лаборатории «Телоэнергетические системы и установки», Выпуск 8, Ульяновск : УлГТУ, 2010, 274 с

[3] T.A. Aliev, N.F. Musaeva, "An algorithm for Eliminating Microerrors of Noise in the Solution of Statistical Dynamics Problems", Automation and Remote Control: Springer, 1998, vol. 59, No. 5, p. 679-688.

[4] N.F. Musaeva, "Robust correlation coefficients as initial data for solving a problem of confluent analysis", Automatic Control and Computer Sciences: Springer, 2007, vol. 41, No. 2, p. 76-87.

[5] N.F. Musaeva, "Robust method of estimation with "contaminated" coarse errors", Automatic Control and Computer Sciences: Springer, 2003, No. 6, p. 50-63.

[6] T.A. Aliev, N.F.Musaeva, M.T.Suleymanova, B.I. Gazizade, "Analytic representation of the density function of normal distribution of noise", Journal of Automation and Information Scien $\neg$ ces: Begell House, 2015, vol. 47(8), No 4, p.2 4-40

[7] T.A. Aliev, N.F.Musaeva, M.T.Suleymanova, B.I Gazizade, "Technology for calculating the parameters of the density function of normal distribution of the useful component in a noisy process", Journal of Automation and Information Scien $\neg$ ces: Begell House, 2016, vol. 48, No 4, p.35-55

[8] T.A. Aliev, N.F.Musaeva, M.T.Suleymanova, B.I. Gazizade, "Digital algorithms for calculating the differential function of normal distribution of noise", National Academy of Sciences of Azerbaijan. Reports, 2016, vol. 72, No 1, p.18-22.

[9] Т.А.Алиев, Н.Ф.Мусаева, М.Т.Сулейманова, Б.И. Газызаде, "Чувствительные алгоритмы выявления степени развития неисправности штанговой глубинной насосной установки", Мехатроника, автоматизация, управление: Москва, 2017, vol, 18, № 2, c. $94-102$

[10] Т.А.Алиев, Н.Ф.Мусаева, М.Т. Сулейманова, “Функция плотности распределения помехи как индикатор для выявления степени развития неисправности штанговой глубинной насосной установки", Проблемы управления и информатики: Киев, 2017, № 2, с. 94-103.

[11] Е.С.Вентцель, Л.А. Овчаров, Теория случайных процессов и ее инженерные приложения. 5-е изд., М. : КНОРУС, 2013, 448 с.

[12] Техническая кибернетика. Книга 2. / Под ред. Солодовникова В.В., М.: Машиностроение, 1967, 682 с. 